\title{
Evaluation of $\alpha$-Amylase Activity and Falling Number around Maturity for Soft White and Soft Red Wheat Varieties in Michigan
}

\author{
N. Yu ${ }^{1,4}$, R. LAurenz ${ }^{1}$, L. Siler ${ }^{1}$, P.K.W. NG ${ }^{2}$, E. SouZA ${ }^{3,5}$ and J.M. LewiS ${ }^{1,5 *}$ \\ ${ }^{1}$ Department of Plant, Soil and Microbial Sciences, Michigan State University, East Lansing, MI 48824, USA \\ ${ }^{2}$ Department of Food Science and Human Nutrition, Michigan State University, East Lansing, \\ MI 48824, USA \\ ${ }^{3}$ Soft Wheat Quality Laboratory, USDA-ARS, Wooster, OH 44691, USA \\ ${ }^{4}$ Current address: Department of Crop Sciences, University of Illinois at Urbana-Champaign, \\ Urbana, IL 61801, USA \\ ${ }^{5}$ Current address: Bayer CropScience LP, Lincoln, NE 68521, USA \\ (Received 31 December 2014; Accepted 10 February 2015; \\ Communicated by F. Békés and A. Aniol)
}

White wheat is, categorically, more susceptible to pre-harvest sprouting (PHS) than red wheat. Physiological maturity (PM), defined as when the seeds reach their maximum dry weight, is a critical time before harvesting. The objective of this study was to determine a reference level of $\alpha$-amylase activity and the corresponding Falling Number (FN) value near the time of PM of selected red and white cultivars in the absence of PHS inducing conditions. Twenty-four soft winter wheat genotypes (12 red and 12 white) adapted to Michigan with varying historic levels of susceptibility to PHS were planted in an $\alpha$-lattice design in two locations from 2008 to 2010. Spikes were collected three days before PM, at PM, and three days post PM. Samples were freeze-dried, threshed, milled and evaluated for $\alpha$-amylase activity and FN value using high throughput method. Within genotype, clear trends were observed in the reduction of $\alpha$-amylase activity and the increase of FN value during the physiological maturation. A nonlinear relationship between $\alpha$-amylase activity and FN value was fit with an $r^{2}$ of 0.801 . Significant differences were observed for genotype for both $\alpha$-amylase activity and FN value for all collection time points. No significant differences were found between red and white wheat, categorically, at any of the three time-points in the absence of PHS. The evaluation results provide a critical reference prior to induction of PHS. The $\alpha$-amylase activity and FN tests show different advantages in analyzing PHS samples as the relationship between $\alpha$-amylase activity and FN value is not linear over wide-ranging results.

Keywords: falling number, wheat, $\alpha$-amylase, Michigan, pre-harvest sprouting, PHS, red wheat, white wheat, quality, breeding

Abbreviations: PHS, pre-harvest sprouting; PM, physiological maturity; FN, Falling Number; DAA, days after anthesis; GDD, growing degree days

\footnotetext{
*Corresponding author; E-mail: janet.lewis@bayer.com
} 


\section{Introduction}

Pre-harvest sprouting (PHS) in wheat causes a sequence of physiological processes, including an increased synthesis and secretion of $\alpha$-amylase and proteases (Gale and Lenton 1987). Starches are hydrolyzed by $\alpha$-amylase and flour quality is lowered. PHS is associated with inadequate seed dormancy, which is a multigenic trait contributed to by the three homoeologous $R$ genes (which determine seed coat color) and regulated by a series of plant growth regulators, of which abscisic acid (ABA) is key (Gale and Lenton 1987; Flintham 2000). Red wheat tends to be more resistant to PHS than white wheat, which may be due to an increased sensitivity of embryos to ABA (Himi et al. 2002). However, for many foods that incorporate bran, a white seed coat is often preferred.

Various methods are used to evaluate PHS and grain dormancy. Visual methods include 'sprout score', an assessment of sprouted seeds in the head (Humphreys and Noll 2002; Munkvold et al. 2009), and 'germination index', a calculation of the percent and speed of seeds germinated outside of the head after moisture is applied (Walker-Simmons and Sesing 1990). The Hagberg Falling Number test measures the functional integrity of the starch structure and is the AACCI standard method for sprouting-damaged cereals evaluation (Hagberg 1960; AACC International 2002). $\alpha$-Amylase activity can also be quantified directly using a chromogenic method (McCleary and Sheehan 1987). Among these diverse evaluation approaches, the visual sprout count method has been reported to be a poor indicator of PHS damage, while $\alpha$-amylase activity and FN tests could clearly distinguish the different baking test samples (Moot and Every 1990). The visual methods are also confounded by germination inhibitors and spike morphology (King and Richards 1984; Kato et al. 2002). Furthermore, enzymatic damage to the starch occurs prior to the visual sprout damage; thus, identification and use of a method which can assess PHS at an earlier stage is warranted for flour quality purpose.

Physiological maturity (PM) is the time when the seed reaches its maximum dry weight and occurs very close to the time when there is a complete loss of chlorophyll color from the glumes (Hanft and Wych 1982; Falcinelli and Giannoni 1985). In the wheat seed development, the germination ability could be formed at the fifth day after flowering and reach the peak after the PM (Gao et al., 2013). Dormancy is peaked at PM and gradually lost during the dry after ripening stage (Paterson et al., 1989). Using glume color to identify the time of PM in wheat has become an effective approach in field evaluation (Humphreys and Noll 2002; Hughes et al. 2010).

The objective of this work was to determine if, in the absence of PHS, there are significant differences in the baseline $\mathrm{FN}$ value and $\alpha$-amylase activity considering grain color (red vs. white) and also within grain color categories around seed PM. The performance of wheat varieties in the absence of PHS is fundamental to understanding the change in FN value and $\alpha$-amylase activity upon induction of PHS. The study reported here links the behavior of FN value and $\alpha$-amylase activity to details about specific genotypes that have been used extensively as breeding parents in the soft wheat region in Eastern North America, which provides great value for breeders, farmers and industry users. 


\section{Materials and Methods}

\section{Plant materials and field design}

In 2008, 10 soft white and 10 soft red winter wheat cultivars adapted to growing in Michigan, USA were selected based on historical visual evaluation of PHS, such that a range of PHS resistance levels was represented in both red and white wheat. In 2009 and 2010, two soft white and two soft red winter wheat cultivars were added to the trials. Field trials were planted in plots with a three-replication $\alpha$-lattice design at each location. Trials were conducted in Clarksville, MI in 2008, East Lansing, MI in 2008 and 2009, Saginaw County, MI in 2009 and 2010, and Lenawee County, MI in 2010.

\section{Physiological maturity determination and sampling}

Flowering time was recorded as the time at which anthers were extruded for $50 \%$ of the spikes in a plot. As each plot began to senesce, three maturity time points were estimated: three days prior to PM (PM-3), PM and three days after PM (PM+3). The maturity time were determined by visualization of the glume chlorophyll loss within the plot as follows: PM-3 $=60 \%$ of heads in the plot had lost their chlorophyll; PM $=80 \%$ of the heads had lost their chlorophyll; PM+3 = three calendar days passed the occurrence of PM. At each time points, 100 spikes were sampled from each plot. The samples were shipped on ice and stored in a freezer $\left(-20^{\circ} \mathrm{C}\right)$ to preserve enzyme levels until they could be freezedried. All spike samples were freeze-dried in either Genesis ${ }^{\circledR}$ 12EL (The Virtis Company, Gardiner, NY) or Tri-Philizer TM MP, (FTS Systems Inc, Stone Ridge, NY). After threshing and cleaning, whole-grain meal was obtained from approximately $40 \mathrm{~g}$ sample of grain using a UDY ${ }^{\circledR}$ Mill (UDY Corp., Fort Collins, CO) with a $0.5 \mathrm{~mm}$ sieve.

\section{Determination of $\alpha$-amylase activity and Falling Number value}

$\alpha$-Amylase activity was measured according to AACCI Approved Method 22-02.01 (Ceralpha method) (AACC International 2002) with a modified protocol developed in USDA Soft White Wheat Quality Lab (Wooster, OH) for high throughput analysis. Twenty $\mu \mathrm{L}$ aliquots of Ceralpha substrate (BPNPG7) solution were dispensed into each well of a 96 well plate and pre-incubated at $42{ }^{\circ} \mathrm{C}$. Three $\mathrm{g}$ of flour sample and $20 \mathrm{~mL}$ of extraction buffer were mixed and incubated at $42{ }^{\circ} \mathrm{C}$ for $20 \mathrm{~min}$, and then centrifuged at $1,500 \mathrm{~g}$ for $15 \mathrm{~min}$ at $35{ }^{\circ} \mathrm{C}$. Twenty $\mu \mathrm{L}$ of $\alpha$-amylase extract was then directly added to the bottom of the well and $300 \mu \mathrm{L}$ of the stopping reagent was added after $20 \mathrm{~min}$. The enzyme reaction for each group of three aliquots pipetted was controlled in 30 seconds intervals to keep the consistent reaction time for the whole plate. The absorbance of each well was read from a microplate reader (BioTek Instruments Inc., Winooski, VT) at $400 \mathrm{~nm}$, using distilled water as control. The enzyme extract was diluted if the absorbance value was greater than 1.2 to avoid substrate saturation. The extinction coefficient $\left(\mathrm{E}_{\mathrm{mM}}\right)$ was determined with a dilution series of $p$-nitrophenol standard solution in $1 \%$ tri-sodium phos- 
phate. Three replications were conducted for each sample. The enzyme activity is presented in Ceralpha units (CU/g).

Flour moisture content was determined using air oven method (AACC International 2000) in 2009 and Near-infrared spectrometer (Bruker Optics Inc., Billerica, MA) in 2010 and 2011. The appropriate amount of flour weight necessary for each FN test was calculated based on $7.0 \mathrm{~g}$ of flour at $14 \%$ moisture. According to AACCI Approved Method 56-81.03 (AACC International 2002), two replications of the FN test were performed on each sample.

\section{Statistical analysis}

Data were analyzed using SAS statistical software 9.2 (SAS Institute, Cary, NC). Analysis of variance (ANOVA) was carried out to using the mixed model where genotype, maturity date and their interaction as fixed effects and year, location, replication, incomplete block as random effects. Fisher's protected Least Significant Difference (LSD) was calculated for each maturity category, for pairwise comparisons at a significance level of 0.05 . All data points for $\alpha$-amylase activity and $\mathrm{FN}$ value were fitting into linear and nonlinear regression models to assess the correlation.

\section{Results}

\section{Environmental conditions}

Across three years, there was no substantial precipitation in the fields during grain-filling (flowering to PM). Average precipitation during that period was less than $11.4 \mathrm{~cm}$ and mean temperature ranged from $18{ }^{\circ} \mathrm{C}$ to $23{ }^{\circ} \mathrm{C}$. No visible sprouting was observed over the three years of the experiment. The average time interval between planting date to PM

Table 1. Two-way ANOVA for the effects of genotype and maturity on the $\alpha$-amylase activity and FN value

\begin{tabular}{|l|r|r|r|r|r|r|}
\hline \multirow{2}{*}{ Source of variation } & \multicolumn{3}{c|}{$\alpha$-amylase activity } & \multicolumn{3}{c|}{ FN value } \\
\cline { 2 - 7 } & df & MS & $F$ & df & MS & $F$ \\
\hline Genotype & 23 & 0.080 & $6.16^{* *}$ & 23 & 7,970 & $10.66^{* *}$ \\
\hline Maturity & 2 & 5.866 & $452.47^{* *}$ & 2 & 841,070 & $1,124.57^{* *}$ \\
\hline Genotype $\times$ maturity & 46 & 0.039 & $2.97^{* *}$ & 46 & 1,230 & $1.64^{*}$ \\
\hline Year & 2 & 0.057 & $2.77^{\text {ns }}$ & 2 & 7,199 & $1.53^{\text {ns }}$ \\
\hline Location (year) & 3 & 0.021 & $2.91^{\text {ns }}$ & 3 & 4,922 & $2.87^{\text {ns }}$ \\
\hline Replication (year $\times$ location) & 10 & 0.007 & $0.60^{\text {ns }}$ & 10 & 1,700 & $1.82^{\text {ns }}$ \\
\hline Block (year $\times$ location $\times$ replication) & 86 & 0.012 & $0.95^{\text {ns }}$ & 86 & 919 & $1.23^{\text {ns }}$ \\
\hline Error & 893 & 0.013 & & 893 & 748 & \\
\hline Total & 1,065 & & & 1,065 & & \\
\hline
\end{tabular}

$* P<0.01 ; * * P<0.0001 ;$ ns Not significant. 
for the soft red and soft white winter cultivars over years and locations was approximately 268 days and an average of 31.5 calendar days after anthesis (DAA) was required for both red and white wheat to reach PM.

\section{$\alpha$-Amylase activity and $F N$ value}

The two way ANOVA test indicated that the main effects of genotype, maturity (PM-3, $\mathrm{PM}, \mathrm{PM}+3$ ) and their interaction were all significant at $p<0.01$ level in the three years $\alpha$-amylase activity and FN study (Table 1 ). The random effects of year, replication and block were not significant at $p<0.01$. When experimental lines were categorized by grain color (red vs. white), only the fixed effect of maturity was significant $(p<0.01$, data not shown). All 24 cultivars showed a decreasing trend of $\alpha$-amylase activity from PM-3 to $\mathrm{PM}+3$, and the degree of decrease between PM-3 to the time of PM was far greater than from $\mathrm{PM}$ to $\mathrm{PM}+3$. There were significant differences among the 24 cultivars for $\alpha$-amylase activity at each of the three time points, even though $\alpha$-amylase activity levels converged towards similar values at PM+3 for all 24 genotypes. For FN, all 24 cultivars increased in FN value from $\mathrm{PM}-3$ to $\mathrm{PM}+3$. In contrast to the converging trend observed over time for $\alpha$-amylase activity, no strong convergence of values was observed for FN test. When examining the red and white cultivars categorically, similar trends were observed.

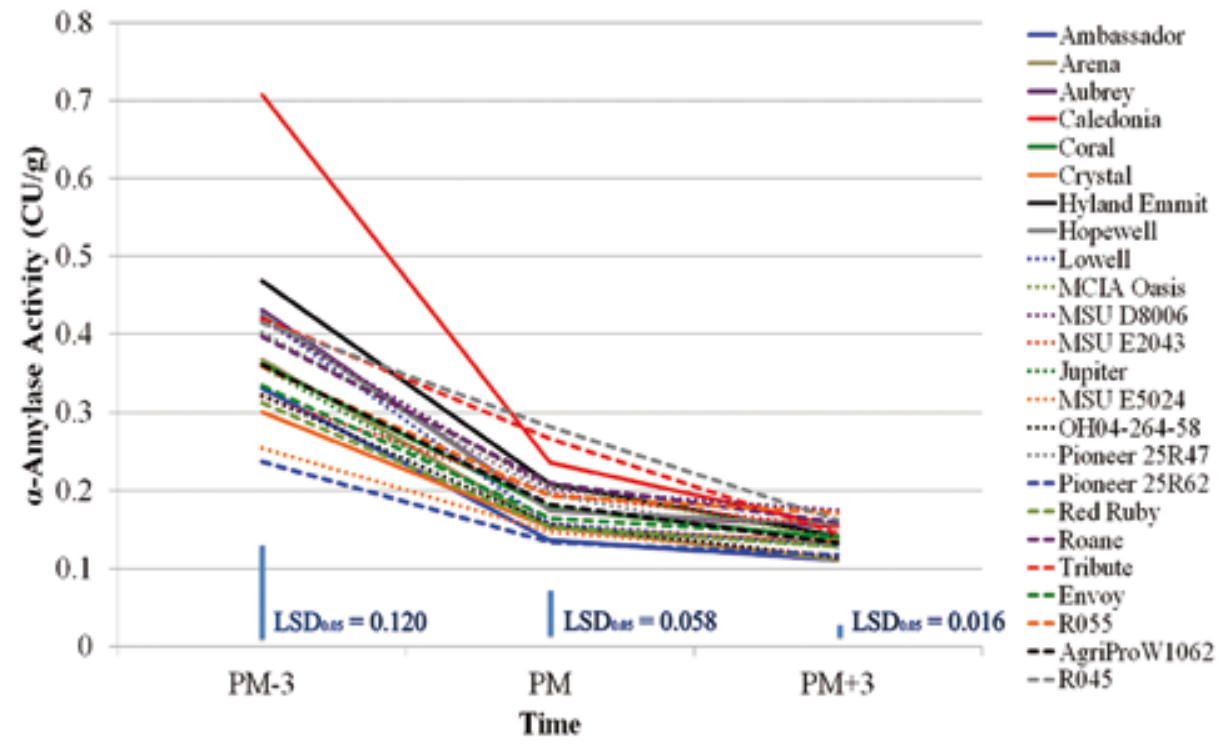

Figure 1. The $\alpha$-amylase activity (CU/g) at three days before physiological maturity (PM-3), PM and three days after PM $(\mathrm{PM}+3)$ for 24 Michigan adapted genotypes, measured from the grain frozen immediately after harvesting. Least square difference (LSD) values $(\alpha=0.05)$ are included at each time point and represented as the vertical bar for comparison 


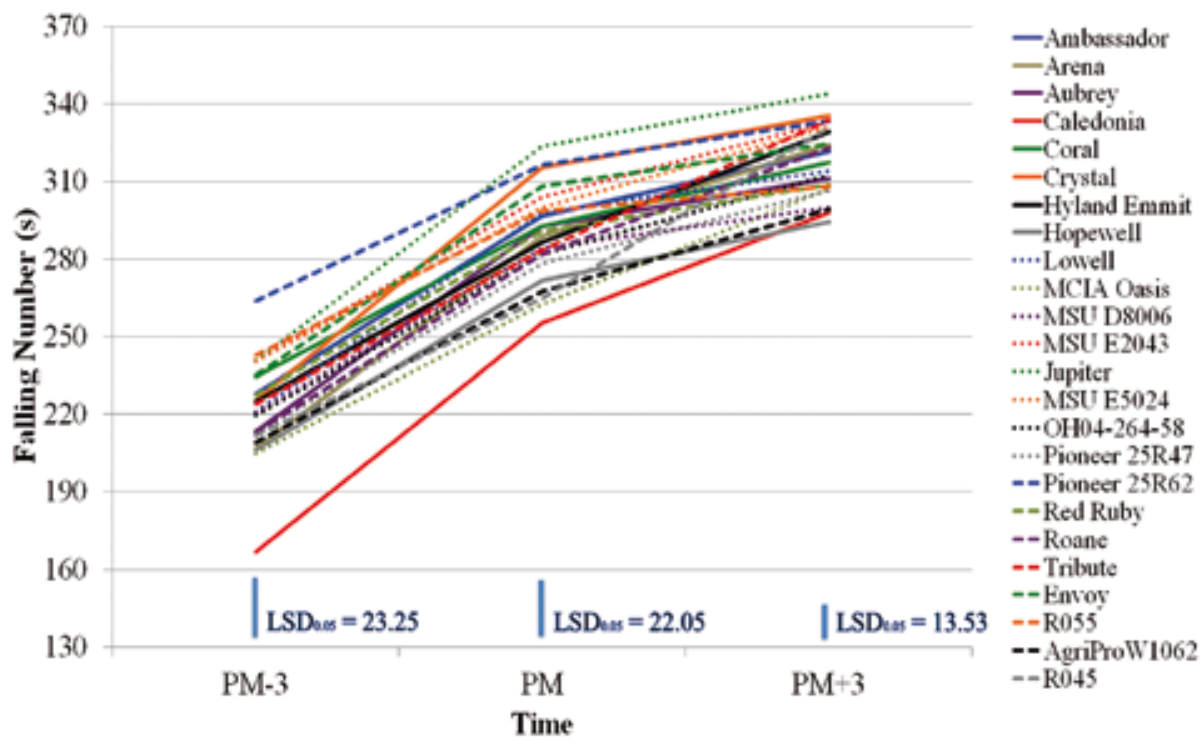

Figure 2. Falling Number value at three days before physiological maturity (PM-3), PM and three days after PM (PM+3) for 24 Michigan adapted genotypes, measured from grain frozen immediately after harvesting. Least square difference (LSD) values $(\alpha=0.05)$ are included at each time point and represented as the vertical bar for comparison

Pairwise comparisons were performed at three time points around PM to determine significant differences between genotypes for both $\alpha$-amylase activity and FN value. For $\alpha$-amylase activity the LSDs were $0.120 \mathrm{CU} / \mathrm{g}, 0.058 \mathrm{CU} / \mathrm{g}$, and $0.016 \mathrm{CU} / \mathrm{g}$ at PM-3, PM and PM+3, respectively ( $\alpha=0.05$ ) (Fig. 1). For FN, the LSDs were $23.25 \mathrm{~s}, 22.05 \mathrm{~s}$, and $13.53 \mathrm{~s}$ at PM-3, PM and PM+3, respectively $(\alpha=0.05)$ (Fig. 2).

\section{Correlation between $\alpha$-amylase activity and $F N$ value}

Both linear and non-linear regression models were evaluated to fit the relationship between $\alpha$-amylase activity and FN value. The coefficient of determination $\left(r^{2}\right)$ of linear regression for $\alpha$-amylase activity and FN value was 0.5743 and the $r^{2}$ for red and white wheat categorically were 0.5216 and 0.6881 , respectively (data not shown). Among the tested non-linear regression models, the best one identified was applied and the $r^{2}$ was 0.801 and root mean square of error (RMSE) was 0.075 .

$$
\alpha \text {-amylase activity }=16098 \times F N^{-0.200}
$$

The predicted $\alpha$-amylase activity and measured $\alpha$-amylase activity versus corresponding FN value in this experiment are shown in Figure 3. Overall, low FN value (below 250s) corresponded with a wide range of $\alpha$-amylase activity value, while little variation was 


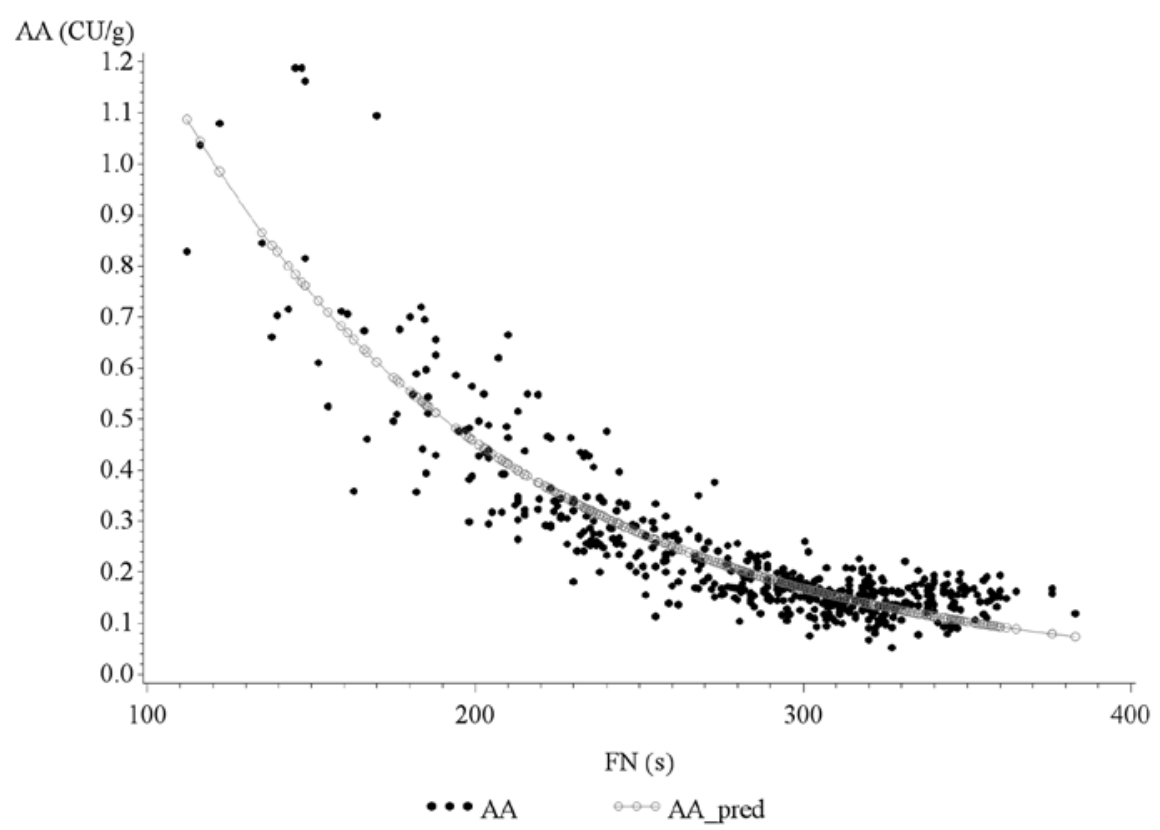

Figure 3. Non-linear relationship between $\alpha$-amylase activity (AA, CU/g) and Falling Number value (FN, s), black dots depict true values of AA and gray circles represent the estimated values for AA by FN

observed in $\alpha$-amylase activity at high FN value. By solving the above equation with natural $\log$ transformation, the relationship between $\alpha$-amylase activity $(\ln )$ and $\mathrm{FN}(\ln )$ is linear:

$$
\ln (\alpha \text {-amylase activity })=9.67-1.9955 \ln F N
$$

\section{Discussion}

In the absence of PHS conditions, significant differences in $\alpha$-amylase activity and FN value were observed among the 24 cultivars. However, unexpectedly, when comparing red wheat and white wheat, differences in $\alpha$-amylase activity and FN value were not significant at any of the three time points around PM. Red wheat is commonly known to have higher seed dormancy at maturity, as well as more resistance to PHS, than white wheat. However, though the $\alpha$-amylase activity is highly diverse at PM- 3 , the white wheat did not show significantly different enzyme activity compared to red wheat, except one extraordinary variety - Caledonia, which is recognized as a PHS susceptible line (Munkvold et al., 2009). Our results suggested $\alpha$-amylase and FN level around PM in red and white wheat is not categorically different under non-sprouting conditions.

It was clear that, irrespective of genotype, $\alpha$-amylase accumulated in the seeds during the maturation process prior to PM, and decreased steadily as PM was approached and surpassed. The increasing trend of FN values across the maturity categories (PM-3 to 
$\mathrm{PM}+3$ ), which indirectly reflects $\alpha$-amylase activity. These results are also in agreement with a study by De Laethauwer et al. (2013), which showed progressively decreasing $\alpha$-amylase activity in two cultivars until PM. In our study, the highest $\alpha$-amylase activity was observed at three days before PM, which is consistent with seed metabolism and energy consumption being reduced as PM is reached. Over years and varieties, the decreasing enzyme activity approaching PM reflects the contribution to the net starch deposition. The converging trend of low $\alpha$-amylase activity at PM and thereafter suggests reduced overall biological activity as the seeds reach PM.

The decreasing enzyme activity during maturation has been reported to result from two main causes: enzymatic protein degradation and inhibition of the enzyme. Wheat $\alpha$-amylase inhibitor could reduce the activity of $\alpha$-amylase to restrain the seeds germination (Mundy et al., 1984). The $\alpha$-amylase inhibitor gene under wheat genetic background could delay sprouting, which is suggested as a strategy to improve PHS resistance (Xiao et al., 2002). In our study, it is possible that the variability of $\alpha$-amylase activity of 24 genotypes at PM-3 could be associated with $\alpha$-amylase content per se or with $\alpha$-amylase inhibitor, but we did not test to distinguish the two factors.

Further studies need to be conducted to determine if the genetic variation observed in the absence of sprouting is generally associated with the variation in conditions that induce PHS. The rising expression of $\alpha$-amylase gene, $\alpha$-Amyl, was found in one susceptible wheat genotype following PM, although the $\alpha$-amylase activity change is not substantial (De Laethauwer et al., 2013). The significant difference on $\alpha$-amylase activity among 24 genotypes in our study may provide the insight of linking enzyme activity towards PHS susceptibility in future research. Because the $\alpha$-amylase activity is highly converged at $\mathrm{PM}$ and $\mathrm{PM}+3$ in our study, these two time points (especially $\mathrm{PM}+3$ ) are expected to be good starting time points to evaluate the effects of inducing sprouting via artificial misting - such that genotypes have a similar "baseline" starting value.

Research by Souza et al. (2011) showed that a high linear correlation between $\alpha$-amylase activity and FN is only present when FN values were between 200 s and 300 s, and two regression functions to fit non-linear relationship between $\alpha$-amylase activity and FN were proposed. In our study, non-linear regression explained the relationship between $\alpha$-amylase activity and FN much better than linear regression, especially when the measured values ranged widely. This relationship was also in agreement with the study by Verity et al. (1999) and Dennett et al. (2013).

From the relationship between $\alpha$-amylase activity and FN, significant reduction in FN could occur without a marked increase in $\alpha$-amylase activity when $\alpha$-amylase activity was low. It has been suggested that FN should not be used as the only method to detect the PHS damage because it does not provide quantified and accurate protein composition and quality changes due to weathering (Barbeau et al., 2006). In addition, Ross et al. (2012) found grain protein concentration could be responsible for changing FN in the absence of elevated $\alpha$-amylase in soft white wheat. The $\alpha$-amylase activity test and FN test show different advantages in analyzing PHS samples as the relationship between $\alpha$-amylase activity and FN is not linear over wide-ranging results. The $\alpha$-amylase levels are highly converged soon after PM during non-PHS inducing conditions, and there is no "upper limit" 
of $\alpha$-amylase values compared to the saturation limit (62s) for FN test. In addition, the high throughput modified Ceralpha method is efficient for screening a large number of samples, as would occur in a breeding program. In contrast, it can be argued that the FN test may be more useful for industry purposes. The FN test shows a high level of variation between genotypes in the absence of sprouting irrespective of time-point around PM, which can reflect different functional properties of the grain. The FN test can raise awareness of when sprouting has occurred, but is not as effective in differentiating levels of sprouting amongst sprouted samples.

\section{Acknowledgments}

This work was supported by Eastern Soft White Wheat Endowment Committee, Michigan State Miller's Association and Michigan Crop Improvement Association.

\section{References}

American Association of Cereal Chemists, International. 2002. Approved Method of AACCI, 10th edition. St Paul, MN, USA.

Barbeau, W.E., Griffey, C.A., Yan, Z.H. 2006. Evidence that minor sprout damage can lead to significant reductions in gluten strength of winter wheats. Cereal Chem. 83:306-310.

De Laethauwer, S., De Riek, J., Stals, I., Reheul, D., Haesaert, G. 2013. Alpha-Amylase gene expression during kernel development in relation to pre-harvest sprouting in wheat and triticale. Acta Physiologiae Plantarum 35:2927-2938.

Dennett, A.L., Wilkes, M.A., Trethowan, R.M. 2013. Characteristics of modern triticale quality: The relationship between carbohydrate properties, $\alpha$-amylase activity, and Falling Number. Cereal Chem. 90:594-560.

Falcinelli, M., Giannoni, G.M.B. 1985. Physiological maturity and visual spike colour in four Italian wheat cultivars. Genetica Agraria 39:283-292.

Flintham, J.E. 2000. Different genetic components control coat-imposed and embryo-imposed dormancy in wheat. Seed Sci. Res. 10:43-50.

Gale, M.D., Lenton, J.E. 1987. Pre-harvest sprouting in wheat: a complex genetic and physiological problem affecting bread-making quality of UK wheat. Aspects of Appl. Biol. 15:115-124.

Gao, X., Hu, C.H., Li, H.Z., Yao, Y.J., Meng, M., Dong, J., Zhao, W.C., Chen, Q.J., Li, X.Y. 2013. Factors affecting pre-harvest sprouting resistance in wheat (Triticum aestivum L.): a review. The J. of Animal \& Plant Sci. 23:556-565.

Hagberg, S. 1960. A rapid method for determining a-amylase activity. Cereal Chem. 37:218-222.

Hanft, J.M., Wych, R.D. 1982. Visual indicators of physiological maturity of hard red spring wheat. Crop Sci. 22:584-588.

Himi, E., Mars, D.J., Yanagisawa, A., Noda, K. 2002. Effect of grain colour gene $(R)$ on grain dormancy and sensitivity of the embryo to abscisic acid (ABA) in wheat. J. Exp. Bot. 53:1569-1574.

Hughes, K.R., Griffey, C.A., Parrish, D.J., Barbeau, W.E., Souza, E., Thomason, W.E. 2010. Preharvest sprouting tolerance in current soft red winter wheat cultivars. Crop Sci. 50:1449-1457.

Humphreys, D.G., Noll, J. 2002. Methods for characterization of preharvest sprouting resistance in a wheat breeding program. Euphytica 126:61-65.

Kato, T., Saito, N., Kashimura, K., Shinohara, M., Kurahashi, T., Taniguchi, K. 2002. Germination and growth inhibitors from wheat (Triticum aestivum L.) husks. J. of Agric. Food and Chem. 50:6307-6312.

King, R.W., Richards, R.A. 1984. Water uptake in relation to pre-harvest sprouting damage in wheat: ear characteristics. Austr. J. of Agric. Res. 35:327-336.

McCleary, B.V., Sheehan, H. 1987. Measurement of cereal $\alpha$-amylase: a new assay procedure. J. of Cereal Sci. 6:237-251. 
Moot, D.J., Every, D. 1990. A comparison of bread baking, Falling Number, $\alpha$-amylase assay and visual method for the assessment of preharvest sprouting in wheat. J. of Cereal Sci. 11:225-234.

Mundy, J., Hejgaard, J., Svendsen I. 1984. Characterization of a bifunctional wheat inhibitor of endogenous $\alpha$-amylase and subtilisin. FEBS 587:995-1268.

Munkvold, J.D., Tanaka J., Benscher, D., Sorrells, M.E. 2009. Mapping quantitative trait loci for preharvest sprouting resistance in white wheat. Theor. Appl. Genet. 119:1223-1235.

Paterson, A.H., Sorrells, M.E., Obendorf, R.L. 1989. Methods of evaluation for preharvest sprouting resistance in wheat breeding programs. Can. J. of Plant Sci. 69:681-689.

Ross, A.S., Flowers, M.D., Zemetra, R.S., Kongraksawech, T. 2012. Effect of grain protein concentration on Falling Number of ungerminated soft white winter wheat. Cereal Chem. 89:307-310.

Souza, E., Costa, J.M., Kratochvill, R. 2011. Falling Number research on wheat. USDA-ARS Soft Wheat Qualty Laboratory. Research Review. Wooster, Ohio, USA. pp. 66-70.

Verity, J.C.K., Hac, L., Skerritt, J.H. 1999. Development of a field enzyme-linked immunosorbent assay (ELISA) for detection of alpha-amylase in preharvest-sprouted wheat. Cereal Chem. 76:673-681.

Walker-Simmons, M., Sesing, J. 1990. Temperature effects on embryonic abscisic-acid levels during development of wheat grain dormancy. J. of Plant Growth Regulation 9:51-56.

Xiao, S.H., Zhang, X.Y., Yan, C.S., Lin, H. 2002. Germplasm improvement for preharvest sprouting resistance in Chinese white-grained wheat: An overview of the current strategy. Euphytica 126:35-38. 